\title{
Development of freeze dried fruit lassi (Indian fruit yoghurt) powders using D-optimal mixture design
}

\begin{abstract}
D-optimal mixture design with 2 factors at 5 levels was used for blend optimization of two fruit lassi (Indian yoghurt drink) powders. The responses studied were overall acceptability and acidity of the formulations. The lassi: Pine apple juice and lassi: Musk melon pulp ratios selected on the basis of response analysis was 49:51 and $72: 28$ respectively. In both lassi powders $0.15 \%$ Carboxy methyl cellulose and $8.5 \%$ sugar were found to be acceptable. The level of juice/pulp in the blend had significant effect on the quality of lassi powders. Freeze dried fruit lassi powders were analysed for proximate composition, physico-chemical and flow properties. $\mathrm{pH}$, acidity of the reconstituted drinks were estimated. The freeze dried mixes had good colour, light weight and were instantly soluble in water. These products offer a healthful combination of dairy and fruit components with the added advantage of culture organisms and low lactose.
\end{abstract}

Keywords: pineapple, muskmelon, curd, freeze drying, mixture design, flow properties
Volume I Issue 3 - 2015

\author{
Shiby Varghese K, Pandey MC \\ Department of Freeze drying and Animal Products Technology, \\ Defence Food Research Laboratory, India
}

Correspondence: Shiby Varghese K, Freeze drying and Animal Products Technology Section, Defence Food Research Laboratory, Siddharthanagar Mysore-5700 I I, India, Tel +91-82I2473425, Fax+9I-82I-2473468, Email shibyk@gmail.com

Received: July 9, 2015 | Published: October 14, 2015

\section{Introduction}

Fermented special products are of interest in the development of new products for the several reasons.. Due to the expanding market of dairy companies, there has been a merging of dairy product and fruit beverage markets, with the introduction of hybrid dairy products, such as 'Juiceceuticals', offering health, flavour and convenience. ${ }^{1}$ Mixed mesophilic cultures of Lactococcus lactis subsp. lactis, Lactococcus lactis subsp. cremoris, Lactococcus lactis subsp diacetylactis along with Leuconostoc species are grown together for production of dahi (Indian Yoghurt). Lassi is a popular and traditional dahi-based drink which originates in the Punjab region. Pineapple (Ananas cosmosus) has been a very popular fruit throughout the world, however in many developing countries only a limited quantity of pineapple products (canned fruit, canned juice or frozen juice concentrate) is produced. During peak season of harvest, as a consequence of poor infrastructure, considerable quantities of fruit can be wasted. ${ }^{2}$ There is an increasing demand for fruit juices with the original characteristics of the fresh fruits and free from chemical additives. This results in the search of new technologies that are able to improve the sensorial, nutritional and microbiological quality of the fruit juices.

Muskmelon (Cucumis melo L.) is one of the economically important fruit in the world and the important quality determining parameters of it are carbohydrates and antioxidants. It is a member of the Cucurbitaceae family. ${ }^{3}$ Consumer preference for this fruit is determined largely by its sweetness (i.e sugar content), flavour or aroma, texture and more recently as a rich source of phytonutrients. ${ }^{4}$ It is rich in ascorbic acid, carotene, folic acid, and potassium as well as a number of other human health-bioactive compounds, vitamin A and vitamin C, and a good source of potassium. ${ }^{5,6}$ It is also a good source of dietary fibre, niacin, vitamin $\mathrm{B}_{6}$ and folate. Musk Melon juice is beneficial to be consumed during conditions like lack of appetite, weight loss, urinary tract infections, constipation, acidity, and ulcer.
Fruit lassi has very low shelf life and hence there is a need to develop process technology for ready to reconstitute shelf stable convenient fruit lassi powders with good retention of antioxidants and vitamins. Although some reports are available on spray dried lassi powders and convective air dried dahi and mango soy fortified yoghurt powder ${ }^{7,8}$ no studies have been carried out on freeze drying of fruit lassi and its quality evaluation. Drying of fruit juices and other products with high sugar content presents technical difficulties because of their hygroscopicity and thermoplasticity at high temperatures and humidities. ${ }^{9}$ For this reason, addition of maltodextrin and gums as well as other substances such as pectin, calcium silicate, and carboxymethyl cellulose, has been used in the production of powder juices. ${ }^{10}$

Statistical design tools such as response surface methodology (RSM) are quite effective in optimizing the ingredient levels in products as well as the process parameters. Basically RSM helps to create a product using regression equations that describe interrelations between input parameters and product properties. ${ }^{11}$ Given a candidate set, the D-optimal design process for mixture designs works exactly the same way as that described for RSM designs. The design points are selected to minimize the variance associated with the estimates of the coefficients in the model you specify. The design space is defined by the low and high level constraints on each factor and any multifactor constraints. D-optimal point selection minimizes the determinant of the $\left(\mathrm{X}^{\prime} \mathrm{X}\right)^{-1}$ information matrix. The optimization is performed to minimize the general variance of the coefficients in the model. D-optimal point selection chooses points from the candidate point set that are spread throughout the design region.

This paper focuses on development of freeze dried pineapple lassi and musk melon lassi powders using D-optimal mixture design and evaluation of their quality characteristics. These powders can be used as instant health drink mixes rich in dairy calcium and fruit antioxidants. The study characterized the physico chemical and flow properties of fruit lassi powders formulated with pineapple and muskmelon with incorporation of $\mathrm{CMC}$, maltodextrin and sugar. 


\section{Materials and methods}

\section{Blend optimisation for fruit lassi preparation}

\section{Juice/pulp extraction}

Musk melon Pulp: Muskmelon (Cucumis melo L.) was procured from local Mysore (India) market. Pulp manufacturing process includes washing, cutting, deseeding, and peeling, refining and finally obtaining pulp through blending in a mixer.

Pineapple juice: Mature giant variety of ripe Kew pineapple was used for this study. Properly ripened pineapple fruits, procured from local Mysore market were peeled, cored, trimmed, and Juice was extracted with a screw juice extractor (model 597 Raylon Metal Works, Mumbai, India) fitted with a 60 -mesh sieve. The resulting juice was pasteurized at $80^{\circ} \mathrm{C}$ for $5 \mathrm{~min}$ in an electrically heated pasteurizer and cooled to room temperature.

Lassi preparation: Lassi was prepared from milk (Nandini Dairy, Mysore) heated to $90^{\circ} \mathrm{C}$ and fermented with mixed mesophilic culture (NCDC -167) propagated using 10\% sterile skim milk as medium. 3\% culture was used and incubated at $30^{\circ} \mathrm{C}$ for $6 \mathrm{~h}$. The curd obtained was mixed in different proportions with pasteurised juice. The flow chart for preparation of fruit lassi is shown in Figure 1.

Experimental design: D-optimal mixture design with no blocking was used for blend optimisation of fruit lassi. The number points in the design were obtained on basis of the number of independent factors (variables) decided for a particular product. The parameters that influenced the product quality, acceptability or functionality and also the shelf life were taken as independent variables. The statistical software package design expert 7.0, Statease Inc., Minneapolis, USA (www.statease.com) was used to construct the experimental design as well as analyse the data. The overall acceptability (OAA) and acidity of the product were selected as the responses. The centre points for these designs were selected keeping the ingredients at levels expected to yield, at least, satisfactory experimental results. The fruit lassi powders obtained were reconstituted before serving to the panel.

The different formulations of the two fruit lassi powders were analysed for the planned responses i.e. sensory score in general as well as acidity. The data for formulations along with responses were analysed using statistical software for the best-fit design and the optimized compositions obtained were further packed and stored for further evaluation.

\section{Freeze drying of the optimised formulation and reconstitution}

Freeze drying: Freeze drying of fruit lassi beverages were carried out in a freeze dryer (Martin Christ, Germany) after adjusting the TSS to $22^{\circ}$ Brix. A vacuum of $0.1 \mathrm{~mm}$ of $\mathrm{Hg}$ was maintained. The freezing temperature was $-40^{\circ} \mathrm{C}$ and secondary heating was carried up to $60^{\circ} \mathrm{C}$. The freeze drying was completed in $16 \mathrm{~h}$ and the flakes were powdered in Raymond mill.

Packing: Each type of fruit lassi powder samples was powdered in a Raymond mill and passed through a 40-mesh sieve to obtain uniform particle size and packed separately in 25-50g bulks using PFP (Paper $45 \mathrm{GSM} /$ Aluminium foil $20 \mu /$ Low-density polyethylene $37.5 \mu$ ) sachets.
Sensory evaluation: The samples were subjected to sensory evaluation on $9 \mathrm{pt}$ hedonic scale by a semi-trained panel of 11 judges. Fruit lassi samples were prepared in $100 \mathrm{ml}$ cups and stored overnight at $2-5^{\circ} \mathrm{C}$ before subjecting them to organoleptic evaluation. The samples were served at $10^{\circ} \mathrm{C}$. A panel of 11 judges, from among the faculty and research scholars of Defence Food Research Laboratory, Mysore was formed on the basis of their knowledge of the product and their willingness to participate on a regular basis after obtaining agreement among the judges on the characteristics of good quality fruit lassi and what they meant by the terminology for sensory evaluation. Appearance and colour, consistency, flavour and overall acceptability were rated on a 9point hedonic scale.

\section{Quality Analyses of powder and reconstituted samples}

Physico- Chemical Analyses: The moisture content of the sample was determined by vacuum oven drying method using constant weight determination method as per. ${ }^{12}$ Acidity, total and reducing sugar content of the samples were determined as per standard procedures used for analysis of fruit products. ${ }^{13}$ Water activity, Bulk density, colour $\left(L^{*} a^{*} b^{*}\right)$ and dispersibility were estimated within $12 \mathrm{~h}$ after drying. Dispersibility of a powder in water is the amount of dry matter which after stirring for 15 seconds with a spoon can pass through a 210 microns sieve. ${ }^{14}$ Thirteen g lassi powder was stirred with $100 \mathrm{ml}$ water at $50^{\circ} \mathrm{C}$ in a beaker for 15 seconds. The reconstituted lassi was then filtered through a 210 micron sieve. The dry matter of the filtered lassi was estimated by the standard method prescribed. ${ }^{12}$ The percent dispersibility was calculated using the Eq.

$$
D s=\frac{(100+13) S p}{13 \times S r}
$$

Where, $\mathrm{Sp}=$ Total solids in lassi powder $(\%)$

$\mathrm{Sr}=$ Total solids in reconstituted lassi powder after it passes through the sieve (\%)

Colour change during drying was evaluated using equation 1

$$
\Delta E c=\sqrt{(L i-L r)^{2}+(a i-a r)^{2}+(b i-b r)^{2}}
$$

Where Li, ai, bi are L, a, b values for beverage samples and $\mathrm{Lr}$, br, ar are the corresponding values for reconstituted powder. Non enzymatic browning of the samples was determined as per the procedure. ${ }^{13}$ About $1-2 \mathrm{~g}$ of the sample was blended with $66 \%$ alcohol and made up to known volume followed by filtration. The OD of the filtrate was measured at $420 \mathrm{~nm}$. Proximate composition was estimated using standard analytical procedures. ${ }^{12}$ Anthocyanins were determined using spectrophotometric method.

Flow characteristics: Powder flowability is the ability of a powder to flow. By this definition flowability is sometimes thought of as a one dimensional characteristics of a powder, where by powder can be ranked on a sliding from free flowing to non flowing. The flowability characteristics of freeze-dried fruit lassi powder like basic flowability energy, conditioned bulk density, compressibility, pressure drop, aeration ratio and aerated energy were determined using FT4 powder rheometer (FT4 Freeman Technology, Bouiters Farm Centre, 2007). 
Viscosities of the reconstituted samples were determined using a viscometer (MCR, Anton Paar Pvt. Ltd. India).

Microbiological quality evaluation: Testing and quantification of coliforms, yeasts and mold was done according to standard methods for examination dairy products (Marth, 1998) using the violet red bile agar (VRBA) and acidified potato dextrose agar (PDA), respectively. Lactic acid bacteria count was done using M17 Agar (Hi media, Mumbai, India).
Statistical analysis: Data were represented as mean of triplicates. One way analysis of variance (ANOVA) using Microsoft Excel was performed according to a factorial design on the basis of complete randomized design (CRD). Duncan's multiple range test (DMRT) was employed to determine the statistical significance $(\mathrm{P}<0.05)$ of the differences among the mean values. Significant differences were indicated by different letters in the Tables 1-3.

Table I Chemical composition of curd, pineapple juice and musk melon pulp

\begin{tabular}{llll}
\hline Chemical composition & Curd & Pineapple juice & Muskmelon pulp \\
\hline Protein & $4 \pm 0.02$ & $0.7 \pm 0.01$ & $0.6 \pm 0.02$ \\
Fat & $3.5 \pm 0.03$ & $0.2 \pm 0.003$ & $0.1 \pm 0.01$ \\
Carbohydrate & $4.7 \pm 0.01$ & $11 \pm 0.04$ & $4.1 \pm 0.1$ \\
Moisture & $85 \pm 1$ & $87 \pm 2$ & $87 \pm 0.1$ \\
Ash & $2.8 \pm 0.02$ & $0.6 \pm 0.03$ & $8.2 \pm 0.01$
\end{tabular}

The values shown are means of 3 replications with standard deviations.

Table 2 Proximate composition and quality parameters of plain lassi powder and fruit lassi powder

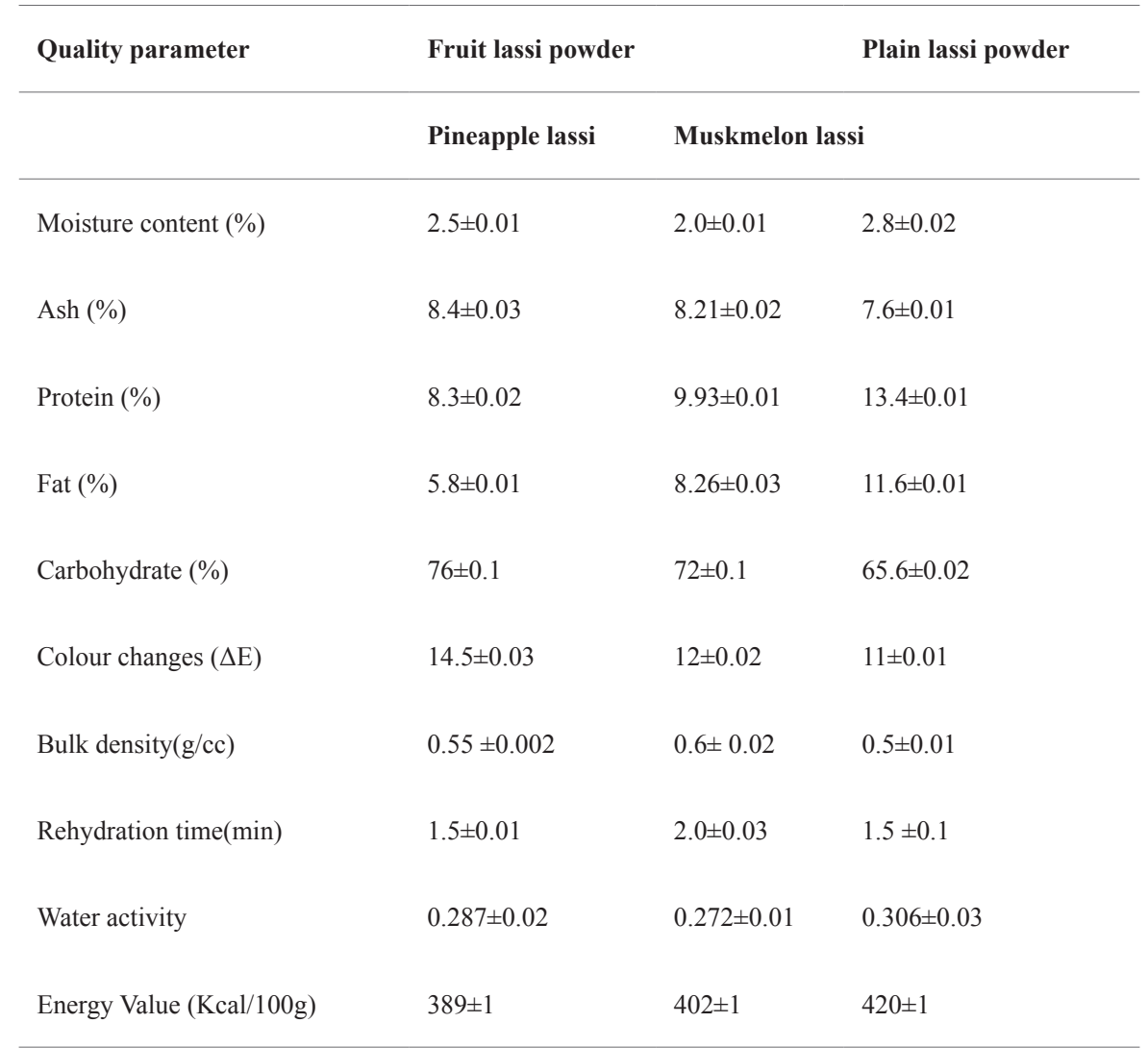

The values shown are means of 3 replications with standard deviations. 
Table 3 Flow properties of pineapple lassi powder and muskmelon lassi powder

\begin{tabular}{lll}
\hline Flowability parameters & Pineapple lassi powder (PLP) & Musk melon lassi powder (MMLP) \\
\hline Basic Flowability Energy BFE $(\mathrm{mJ})$ & $550 \pm 5$ & $817 \pm 6$ \\
Conditioned Bulk Density CBD $(\mathrm{g} / \mathrm{ml})$ & $0.43 \pm 0.02$ & $0.485 \pm 0.01$ \\
Compressibility CPS15(\%) & $21.9 \pm 0.5$ & $21.4 \pm 0.02$ \\
Pressure Drope & $5.64 \pm 0.02$ & $11.0 \pm 0.03$ \\
PD15(mbar) & & \\
Aeration Ratio, AR10 & $4.5 \pm 0.01$ & $9.91 \pm 0.02$ \\
Aerated Energy, AE10 $(\mathrm{mJ})$ & $161 \pm 2$ & $75.7 \pm 3$ \\
\hline
\end{tabular}

The values shown are means of 3 replications with standard deviations.

\section{Results and discussion}

\section{Blend optimisation for fruit Lassi preparation}

The chemical composition of curd, pineapple juice and muskmelon pulp were evaluated and the results are shown in Table 1 . The process flow chart for preparation of fruit lassi powders is shown in Figure 1. The sensory score was selected as one of the responses in D-optimal mixture design for blend optimization of both the products because it is considered to be dependent on specific composition of the products and decide the acceptability while acidity, colour and viscosity of the beverages decide the final taste, appearance, consistency and storage behaviour of these products. The changes in selected responses for both pine apple lassi and musk melon lassi beverages as given by the two component design are shown in Figures $2 \&$ Figure 3. The responses for the D-optimal mixture designs were modelled to fit a second order polynomial equation. RSM in optimization of product ingredients and process variables has also been reported by many researchers. ${ }^{15-19}$

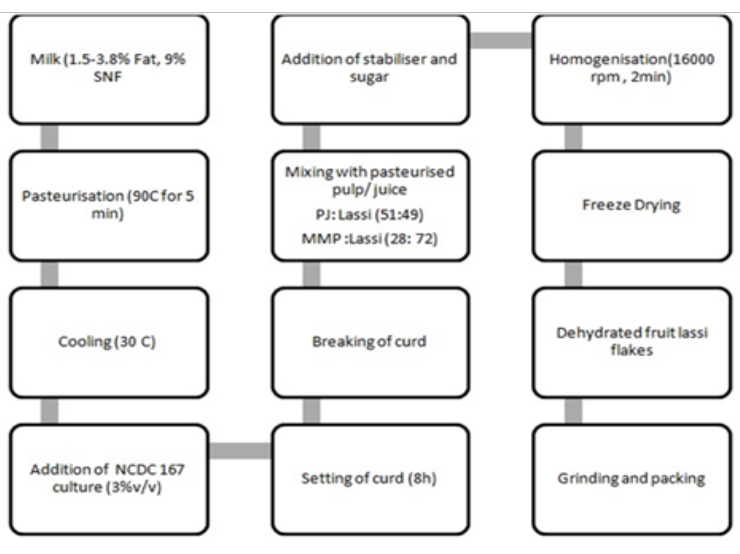

Figure I Process flow chart of freeze dried fruit lassi powder.

The responses of these models can be plotted as a function of two components in the mixture keeping the total as 100 . The variation in overall acceptability with varying contents of lassi and pineapple juice in the blend has been depicted in Figure 2. The common operation in manufacture of fruit lassi is to blend lassi and fruit components followed by proper heating and packaging. To minimize heat induced precipitation of proteins $\mathrm{pH}$ adjustment is necessary. ${ }^{20}$ Acidity of the blend decreased as proportion of muskmelon pulp increased. Reverse trend was observed in case of pineapple juice when blended with lassi. This may be due to the higher acidity of pineapple juice in comparison with the sweet curd used in study. Higher proportions of pine apple juice imparted more tartness and thicker consistency which was not liked by the panel. Samples which contained more of lassi lacked in colour. Higher proportions of musk melon pulp imparted predominant musky taste and thicker consistency which was not preferred by the panel. Musk melon lassi contained more of lassi lacked in colour. Pineapple lassi was found to be more acidic, musk melon lassi was found to be thicker in consistency. In case of pineapple lassi, heat treatment of pineapple juice prior to mixing with lassi was very essential to inhibit the bromelain enzyme and hence to avoid bitterness. Cold mixing of lassi with pineapple juice result in a bitter product which is not sensorily acceptable. The changes in colour $(\Delta \mathrm{E})$ during freeze drying were more pronounced in case of pineapple lassi powder than musk melon lassi powder. Regression equations in terms of coded factors generated for all factors in pineapple lassi and musk melon lassi have been represented as follows

Acidity $=-0.08 * \mathrm{~L}+2.07 * \mathrm{PJ}-0.02 * \mathrm{~L} * \mathrm{PJ}+3.62 * \mathrm{~L} * \mathrm{PJ} *(\mathrm{~L}-\mathrm{PJ}) \ldots \ldots$. $\mathrm{R}^{2}=0.97$

$$
\mathrm{OAA}=+6.83661 * \mathrm{~L}+6.62076 * \mathrm{PJ}+6.33 * \mathrm{~L} * \mathrm{PJ} \ldots \ldots \ldots . . \mathrm{R}^{2}=0.98
$$

A c idity $=+1.93 * \mathrm{~L}-0.36 * \mathrm{M} \mathrm{M}-0.51 * \mathrm{~L} * \mathrm{M} \mathrm{M}-$ $5.12 * \mathrm{~L} * \mathrm{M} \mathrm{M} *(\mathrm{~L}-\mathrm{M} \mathrm{M}) \ldots \mathrm{R}{ }^{2}=0.99$ $\mathrm{OAA}=+7.91 * \mathrm{~L}+6.3 * \mathrm{MM}+3.16 * \mathrm{~L} * \mathrm{MM} \ldots \ldots \ldots \ldots . . . \mathrm{R}^{2}=0.95$

Where PJ is pineapple juice, MM is musk melon pulp and $\mathrm{L}$ is Lassi in the blend

The statistical analysis indicates that the proposed model was adequate, possessing no significant lack of fit and with very satisfactory values of the $\mathrm{R}^{2}$ for all the responses. The equations fitted had high $\mathrm{R}^{2}$ values indicating their good prediction accuracy for overall acceptability and acidity of beverages containing varying levels of lassi and pine apple juice/musk melon pulp. The desirable maximization of the polynomials thus fitted was performed by numerical techniques using mathematical optimization procedure of design expert software package. The criteria set for optimization were maximization of sensory score which is thought to be most important parameter in product development studies. The solutions were obtained using the software, which sought to maximize the desirability function by being at random starting points and proceeding on a path of the steepest slope to a maximum. The best among them was taken as optimum. The optimized ingredient levels of lassi: fruit pulp/juice were 72:28 and 49:51 for musk melon and pineapple respectively. The fruit lassi powders were prepared using the optimised ingredient 
composition. The reconstitution of the mixes was carried out at the rate of $30 \mathrm{~g}$ per $150 \mathrm{ml}$ serving. The predicted responses and actual obtained response values were similar, hence the fitted models are suitable for predicting the responses.

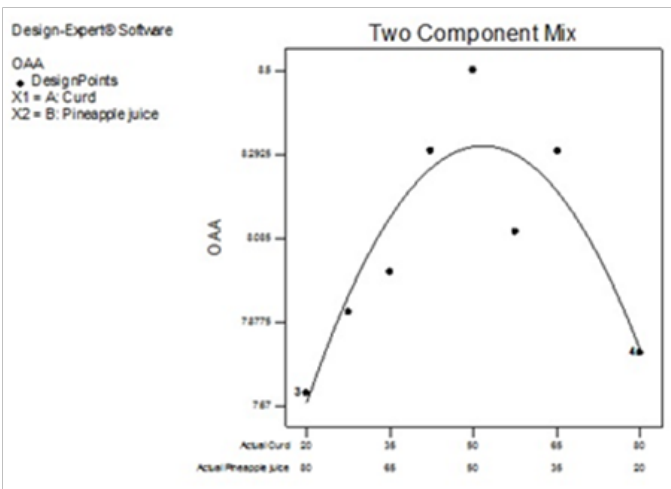

Figure 2 Variaition in Overall acceptability of fruit lassi with varying proportions of lassi and pineapple Juice in the blend.

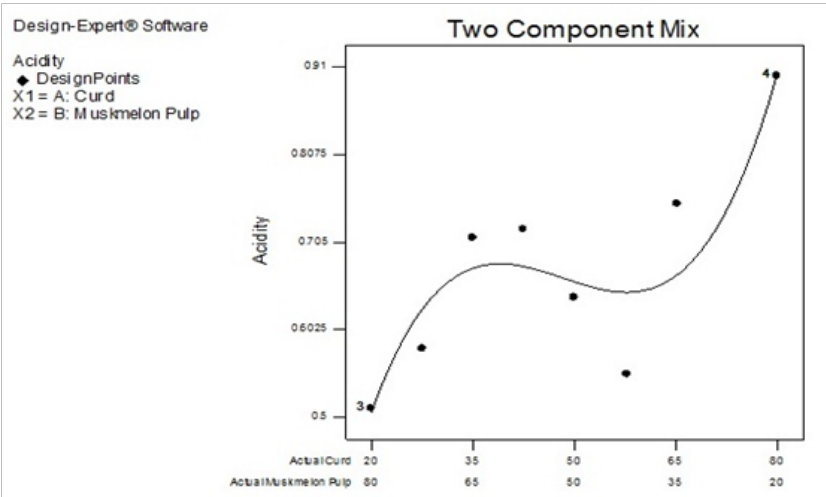

Figure 3 Variation in acidity of fruit lassi with varying proportions of lassi and pineapple juice in the blend.

\section{Quality analyses of powder and reconstituted samples}

Proximate composition and microbiological quality of fruit lassi powders: The proximate composition and physico-chemical quality parameters of the fruit lassi powders and plain lassi powder are shown in Table 2. Reconstituted Pineapple lassi and muskmelon lassi had a calorific value of 116.7 and $120.6 \mathrm{Kcal}$ per serving of $100 \mathrm{ml}$. The dispersibility of the Pineapple lassi powder (PLP) and muskmelon lassi powder (MMLP) are 95 and $94 \%$ respectively. Bulk density is in the order MMLP $>$ PLP $>$ LP. The rehydration time required is slightly higher for MMLP than for PLP. Yeasts and molds and coliforms were nil in freeze dried products initially and also at the end of 10 month storage at ambient conditions.

About $10-25 \%$ pineapple juice obtained from canning industry is not suitable for production of single strength or concentrate juice, due to the high acidity. The present study resulted in an instant drink mix with lower acidity than pure pineapple juice and better nutritional quality due to the incorporation of milk solids in the form of a fermented dairy product with improved digestibility.
Flow properties: Table 3 summarises the flow properties of freeze dried fruit lassi powders obtained with pineapple juice and musk melon pulp. Flowability is an important but complex property controlled by particle size distribution, angle of repose and moisture content of powder. The flowability is usually measured as the time needed for a certain volume of powder to pass through slots of a specified size in a rotating drum. ${ }^{21}$ Basic Flow Energy (BFE )was found to be higher for Muskmelon lassi powder than Pineapple lassi powder. Permeability was inversely correlated with the pressure drop measurements. The two powders tested had similar compressibilities.

Compressibility is a measure of how density changes as a function of applied normal stress. For powders, this bulk property is influenced by many factors such as particle size distribution, cohesivity, particle stiffness, particle shape and particle surface texture. It is not a direct measurement of flowability, but nevertheless relates to many process environments, such as storage in hoppers or super sacks, or behaviour during roller compaction, for example. Generally, cohesive powders consisting of mainly sub $30 \mu$ particle size are the most compressible, with powders of a granular nature being least compressible. Of course there are exceptions to this generalisation and powders consisting of large but very elastic particles may also become readily compressed. Permeability is a measure of how easily air passes through a powder at increasing bulk stress. Close packed, coarse powders have air voids between the particles and hence are usually highly permeable. Cohesive powders tend to have a large amount of entrained air, but voids are not connected so that permeability is poor. The test is usually represented as pressure drop across the powder bed versus normal stress, for a constant air velocity. The greater the pressure drop, the less permeable the powder.

Pineapple lassi has lower pressure drop which implies higher permeability. Pineapple lassi . Powder showed poorer response to the introduction of air and is likely the more cohesive as indicated by its high Aerated Energy and low Aeration Ratio. Measuring mass of a precise volume of conditioned powder provides the Conditioned Bulk Density (CBD). The CBD can be greatly increased by consolidation or conversely, reduced by aeration. The extent of this change being dependent upon whether the powder is cohesive or not and also how much consolidation was produced. The density reported as "poured density", used in calculation of parameters such as Carr's Index and Hausner Ratio. However, poured density is always dependant on the apparatus used and on the way the operator handles the powder. Therefore, high variability in the result is common for techniques that depend on poured density values. The CBD values obtained in our study characterises the flow properties of fruit lassi powders and is a good indication of the cohesiveness and aeration in the powder samples. Sonner et al. ${ }^{22}$ evaluated process effects on the properties of Spray-Freeze-Dried Powders. Bulk densities, compaction densities, and Hausner ratios of the powders were significantly higher in freeze drying method compared with hot air drying method. ${ }^{23}$ Flow properties of food powders and method for evaluation have been reviewed by Peleg. ${ }^{24}$ Hygroscopicity, degree of caking, dispersibility, flowability, sticky point temperature of the vacuum dried mango powder at $5 \%(\mathrm{db})$ moisture content and overall color difference between the reconstituted powder and the pulp were found out. ${ }^{25}$ Due to the absence of any specified range of values for fruit juice powders, the authors measured the above-mentioned properties of commercially available Instant coffee and tomato soup powders. Instant coffee powder was chosen for its high hygroscopicity. The tomato soup powder, being 
made of high molecular weight substances like starch, maltodextrin, 2 corn and wheat flour, had its hygroscopicity lower than the instant coffee powder. It was considered that mango powder would have the desirable quality if its properties lie within the range of properties of 3 . the above-mentioned food powders. Similar assumptions are made for flow property analysis of freeze dried fruit lassi powders.

Temperature has an important role on rheological characteristics of any food product. In both the powders after reconstitution, the viscosity decreased with increasing temperature. Musk melon lassi had higher viscosity than Pineapple lassi in the temperature range studied. The viscosity of reconstituted Muskmelon lassi powder decreased from $40 \mathrm{cps}$ at $5^{\circ} \mathrm{C}$ to $20 \mathrm{cps}$ at $40^{\circ} \mathrm{C}$. There was no further reduction in viscosity by increasing temperature from $40-60^{\circ} \mathrm{C}$. The viscosity of Pineapple lassi powder decreased from $40 \mathrm{cps}$ at $5^{\circ} \mathrm{C}$ to $8 \mathrm{cps}$ at $50^{\circ} \mathrm{C}$. The viscosity values obtained were much lower than those for pure curd ${ }^{26}$ but greater than those for pomelo juice $\left(20^{\circ}\right.$ Brix $)$ which exhibited concentration dependent Newtonian and Non Newtonian behaviour as reported by Keshani et al. ${ }^{27}$ The viscosity values in fruit products are also related to the sugar content. The apparent viscosity was Shiby et al. ${ }^{26}$ reported the viscosity of curd (Indian yoghurt) as a function of starter culture level in the product and the values ranged from $336 \times 10^{3} \mathrm{cps}$ to $512 \times 10^{3} \mathrm{cps}$. Stirred curd has lower viscosity than set curd. The complex viscosity of the matrix may be an indication of the final porosity and other structural properties.

\section{Conclusion}

Two freeze dried fruit lassi powders containing varying proportions of lassi and fruit juice/ pulp have been formulated with the help of D-optimal mixture design. Acidity and colour of the mixes were mainly controlled by the proportion of lassi and fruit juice. The viscosity of reconstituted fruit lassi powders decreased with increase in temperature. Musk melon lassi had higher viscosity than Pineapple lassi and viscosity of both the samples decreased with increasing temperature. Mixture design was found to be beneficial tool in product development with high degree of prediction accuracy for the responses. The fruit lassi powders developed were found to be light weight, more porous structure with minimum shrinkage, instantly soluble and good in colour. The powders also exhibited good flowability, aeration and compressibility characteristics. The freeze dried fruit lassi powders developed are fermented special products which combine the health benefits of lassi with the antioxidants and vitamins from fruit components. These products have improved sensory scores and nutritional quality than plain lassi powder. Freeze dried fruit lassi powders can also be utilised as ready to reconstitute health drink mixes with reduced bulk suitable for combat conditions and high altitudes faced with logistic problems.

\section{Acknowledgements}

Project funding from Defence Research and Development Organisation, India is duly acknowledged.

\section{Conflict of interest}

The author declares no conflict of interest.

\section{References}

1. Khurana HK, Kanawjia SK. Recent trends in development of fermented milks. Current Nutr and Food Sci. 2007;3(1):91-108.
Nicoleti JF, Telis-Romero J, Telis VRN. Air-drying of fresh and osmotically pre-treated pineapple slices: Fixed air temperature versus fixed slice temperature drying kinetics. Drying Technol. 2001;19(9):2175-2191.

3. Bailey LH, Bailey EZ. Hortus Third. A Dictionary of Plants Cultivated in the United States and Canada. New York, USA: Macmillan Publishing Co, Inc; 1976. $330 \mathrm{p}$.

4. Lester GE. Antioxidant, sugar, mineral, and phytonutrient concentrations across edible fruit tissues of orange-fleshed Honeydew melon (Cucumis melo L.). J Agri and Food Chem. 2008;56(10):3694-3698.

5. Lester GE, Hodges DM. Antioxidants associated with fruit senescence and human health: Novel orange fleshed non-netted honey dew melon genotype comparisons following different seasonal productions and cold storage durations. Postharvest Biol and Technol. 2008;48(3):347-354.

6. Menon SV, Ramana Rao TV. Nutritional quality of muskmelon fruit as revealed by its biochemical properties during different rates of ripening. Int Food Res J. 2012;19(4):1621-1628.

7. Kumar P, Mishra HN. Storage stability of mango soy fortified yoghurt powder in two different packaging materials: HDPP and ALP. J Food Eng. 2004;65(4):569-576.

8. Shiby VK, Mishra HN. Modelling of acidification kinetics \& textural properties in buffalo milk curd (Indian yoghurt). Int J Dairy Technol. 2008;61(3):284-289.

9. Adhikari B, Howes T, Bhandari BR, et al. Effect of addition of maltodextrin on drying kinetics and stickiness of sugar and acid-rich foods during convective drying experiments and modelling. J Food Eng. 2004;62(1):53-68.

10. Bhandari BR, Snoussi A, Dumoulin ED, et al. Spray drying of concentrated fruit juices. Drying Technol. 1993;11(5):1081-1092.

11. Colona P, Doublier JL, Melcion JP, et al. Extrusion cooking and drum drying of wheat starch. I. Physical and macromolecular modifications. Cereal Chem. 1984;61:538-543.

12. AOAC. Official method of analysis. 14th ed. Association of official Analytical chemists. USA: Washington D.C; 1990.

13. Ranganna S. Hand book of analysis and quality control for fruit and vegetable products. New Delhi, India: Tata McGraw-Hill Publishing Company Ltd; 1986. p. 9-10 \& 88-94.

14. Haugaard IS, Krag J, Pisecky J. Analytical methods for dry milk powders. Niro Atomizer, Denmark; 1978.

15. Gill BS, Sodhi NS, Narpinder Singh, et al. Effects of o brix, sodium alginate and drying temperature on color, texture and sensory properties of Dushehari mango leather. J Food Sci and Technol. 2004;41(4):278-373.

16. Ismail YS, Rustom, MH, Lopez-levia, et al. Optimization of extraction of peanut proteins with water by response surface methodology. J Food Sci. 1991;56:1660-1663.

17. Raghavan CV, Srichandan BR, Nagin Chand, et al. Response surface analysis of power consumption of dough sheeting as a function of gap, reduction ratio, water, salt and fat. J Food Sci and Technol. 1996;33(4):313321.

18. Rathi P, Goswami VK, Sahai V, et al. Statistical medium optimization and production of a hyperthermostable lipase from Burkholderia cepacia in a bioreactor. J Appl Microbiol. 2002;93(6):930-936.

19. Shiby VK, Radhakrishna K, Bawa AS. Development of whey- fruit based energy drink mixes using D-optimal mixture design. Int J Food Sci and Technol. 2013;48(4):742-748.

20. Jelen P, Buchheim W. Stability of whey protein upon heating in acidic conditions. Milchwissenchaft. 1984;39:215-218. 
21. Pisecky J. Standards specifications and test methods for dry milk products MC Diarmuid editor. Concentration and drying of foods. New York, USA Elsevier Sci Publ Co; 1985. p. 203-220.

22. Sonner C, YF Maa, G Lee. Process effects on the properties of sprayfreeze-dried powders. $3^{\text {rd }}$ World Meeting on Pharmaceutics; 2000.

23. Lee CW, Oh HJ, Han SH, et al. Effects of hot air and freeze drying methods on physicochemical properties of citrus hallabong powders. Food Sci and Biotechnol. 2012;21(6):1633-1639.

24. Peleg M. Flowability of food powders and methods for its evaluation-a review. J Food Process Eng. 1977;1(4):303-328.
25. Jaya S, Das H. Effect of maltodextrin, glycerol monostearate and tricalcium phosphate on vacuum dried mango powder properties. J Food Eng. 2004;63(2):125-134.

26. Shiby VK, Mishra HN. Effect of starter culture level on textural properties of buffalo milk dahi. Egypt J of Dairy Sci. 2012;40(1):15-23.

27. Keshani S, Luqman Chuah A, Russly AR. Effect of temperature and concentration on rheological properties pomelo juice concentrates. Int Food Res J. 2012;19(2):553-562. 